\title{
Mid-infrared GaSb-based resonant tunneling diode photodetectors for gas sensing applications
}

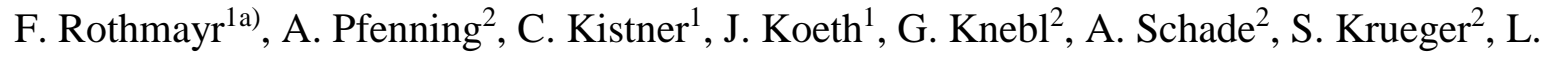

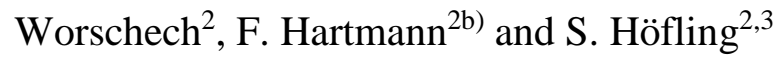 \\ ${ }^{1}$ nanoplus Nanosystems and Technologies GmbH, Oberer Kirschberg 4, D-97218 Gerbrunn, Germany \\ ${ }^{2}$ Technische Physik, Physikalisches Institut and Röntgen Center for Complex Material Systems (RCCM), \\ Universität Würzburg, Am Hubland, D-97074 Würzburg, Germany \\ ${ }^{3}$ SUPA, School of Physics and Astronomy, University of St. Andrews, St. Andrews, KY16 9SS, United \\ Kingdom
}

We present resonant tunneling diode-photodetectors (RTD-PDs) with $\mathrm{GaAs}_{0.15} \mathrm{Sb}_{0.85} / \mathrm{AlAs}_{0.1} \mathrm{Sb}_{0.9}$ double barrier structures combined with an additional quaternary $\mathrm{Ga}_{0.64} \mathrm{In}_{0.36} \mathrm{As}_{0.33} \mathrm{Sb}_{0.67}$ absorption layer covering the fingerprint absorption lines of various gases in the mid-infrared wavelength spectral region. The absorption layer cut-off wavelength is determined to be $3.5 \mu \mathrm{m}$ and the RTD-PDs show peak-to-valley current ratios up to 4.3 with peak current densities of $12 \mathrm{~A} / \mathrm{cm}^{-2}$.The incorporation of the quaternary absorption layer enables the RTD-PD to be sensitive to illumination with light up to the absorption lines of $\mathrm{HCl}$ at $3395 \mathrm{~nm}$. At this wavelength, the detector shows a responsivity of $6.3 \mathrm{~mA} / \mathrm{W}$. At the absorption lines of $\mathrm{CO}_{2}$ and $\mathrm{CO}$ at $2004 \mathrm{~nm}$ and $2330 \mathrm{~nm}$ respectively, the RTD-PD reaches responsivities up to 0.97 A/W. Thus RTD-PDs pave the way towards high sensitive mid-infrared detectors that can be utilized in tunable laser absorption spectroscopy.

\footnotetext{
a) florian.rothmayr@nanoplus.com

b) fhartmann@physik.uni-wuerzburg.de
} 
In their pioneering work more than 40 years ago, R. Tsu and L. Esaki predicted the resonant tunneling of charge carriers in superlattice heterostructures ${ }^{1,2}$. Since then, heterostructures composed of two barriers embedding a single quantum well, double barrier resonant tunneling diodes (RTDs), have been realized and demonstrated in various materials ranging from semiconductors to oxide materials. ${ }^{3,4,5}$ RTDs are especially appealing for high frequency applications due to their relative structural simplicity in combination with a negative differential conductance and high speed electron dynamics, ${ }^{6}$ and RTD oscillators up to the $\mathrm{THz}$ regime have been realized successfully. ${ }^{7,8}$ Apart from the high frequency applications, RTDs are also suitable for opto-electronic applications. RTDs are themselves not only light emitting devices but they can also be utilized as high frequency drivers of semiconductor lasers ${ }^{9}$ or electrically driven devices with an optical bistability. ${ }^{10}$ Furthermore, resonant tunneling diodephotodetectors (RTD-PDs) ${ }^{11}$ covering the visible and near-infrared spectral wavelength region have been demonstrated with light detection capabilities down to the single photon detection threshold. ${ }^{12,13}$ The realization of RTD-PDs operating in the mid-infrared wavelength range between 2 and $4 \mu \mathrm{m}$ is appealing, because several important gases such as $\mathrm{CO}_{2}, \mathrm{CO}$, and $\mathrm{HCl}$ have strong absorption lines $(\lambda=2004 \mathrm{~nm}$, $2330 \mathrm{~nm}$, and $3395 \mathrm{~nm}$ respectively) in this spectral window. ${ }^{14}$ In recent years, research covering this spectral range was driven by both, the emitter and detector part. For tunable laser absorption spectroscopy, single mode emitting diode and interband cascade laser concepts have been realized to cover this spectral region. ${ }^{15,16}$ For the detector part, many different approaches exist that are either temperature or charge controlled, i.e. thermopiles, pyroelectric detectors or photoresistors. ${ }^{17,18}$ These approaches cover a wide range of wavelengths and each concept has its advantages and disadvantages (see Ref. (19) for a comparison between various MIR-sensor concepts). Nowadays, interband cascade detectors (ICDs) receive a considerable amount of technological and scientific interest due to their inherent characteristics, such as low noise and high absorption efficiencies. ${ }^{20,21}$ ICDs are complex devices that require many type I and II heterostructure interfaces, accurate sidewall passivation, and hence demand high accuracy throughout the growth and fabrication processes..$^{22,23}$ In contrast, RTD-PDs are composed of a rather simple heterostructures design. ${ }^{24}$ Moreover, RTD-PDs enable high multiplication factors due to the possibility of controlling the tunneling current via Coulomb interaction of photo-generated minority charge carriers. ${ }^{25}$ In recent publications, we studied the electrical characteristics of RTDs based on $\mathrm{GaSb} / \mathrm{AlAsSb}$ double barrier structures and demonstrated room temperature resonant tunneling by incorporation of ternary GaAsSb or GaInSb prewells. ${ }^{26,27}$ 
In this study, we present resonant tunneling diode photodetectors with a GaAsSb/AlAsSb double barrier structure covering the fingerprint absorption region of various gases in the mid-infrared wavelength region. The RTD-PD utilizes a quaternary GaInAsSb absorption layer with a cut-off wavelength of 3.5 $\mu \mathrm{m}$. At room temperature, peak-to-valley current ratios (PVCR) reach values as high as 4.3. The absorption layer enables light detection up to the absorption lines of $\mathrm{HCl}$ at $3395 \mathrm{~nm}$ with a responsivity of $6.3 \mathrm{~mA} / \mathrm{W}$. Via Coulomb interaction of photo-generated minority charge carriers at the resonant tunneling structure, responsivities up to $0.97 \mathrm{~A} / \mathrm{W}$ are observed for the absorption lines at $2004 \mathrm{~nm}\left(\mathrm{CO}_{2}\right)$.

The sample is grown on an n-type Te doped GaSb (100) substrate via molecular beam epitaxy (MBE) which is equipped with solid-state evaporation cells for the group-III elements and dopants. Group-V elements are provided by valved cracking cells. The bottom contact region consists of $300 \mathrm{~nm}$ and 120 $\mathrm{nm}$ Te n-doped GaSb layers with doping concentrations of $n=2 \times 10^{18} \mathrm{~cm}^{-3}$ and $n=5 \times 10^{17} \mathrm{~cm}^{-3}$, respectively. The undoped resonant tunneling structure (RTS) is formed by a $20 \mathrm{~nm} \mathrm{GaSb}$, a $7 \mathrm{~nm}$ $\mathrm{GaAs}_{0.15} \mathrm{Sb}_{0.85}$ emitter prewell, a $5 \mathrm{~nm}$ thick GaAs${ }_{0.15} \mathrm{Sb}_{0.85}$ quantum well, two $4 \mathrm{~nm}$ thick $\mathrm{AlAs}_{0.1} \mathrm{Sb}_{0.9}$ barriers and $5 \mathrm{~nm} \mathrm{GaSb}$. Thereafter, the quaternary $\mathrm{Ga}_{0.64} \mathrm{In}_{0.36} \mathrm{As}_{0.33} \mathrm{Sb}_{0.67}$ absorption layer is grown. The first $20 \mathrm{~nm}$ are undoped and the following $130 \mathrm{~nm}$ and $50 \mathrm{~nm}$ have doping concentrations of $n=1 \times$ $10^{17} \mathrm{~cm}^{-3}$ and $n=5 \times 10^{17} \mathrm{~cm}^{-3}$, respectively. Subsequently, the top contact region consisting of 150 $\mathrm{nm} \mathrm{GaSb}\left(n=1 \times 10^{18} \mathrm{~cm}^{-3}\right)$ follows and the whole structure is finalized with a $10 \mathrm{~nm} \mathrm{GaSb}$ cap $(n=$ $2 \times 10^{18} \mathrm{~cm}^{-3}$ ). Fig. 1(a) shows a numerical simulation of the RTD-PD bandprofile ( $\Gamma$ and $\mathrm{HH}=$ heavy hole) with the shaded absorption layer and RTS region which are embedded in the n-doped GaSb contact regions. The numerical simulations are performed with the nextnano tool. ${ }^{28}$ The mole fractions of the quaternary absorption layer are designed for a bandgap energy corresponding to a cut-off wavelength of $3.5 \mu \mathrm{m}$. The transition energy spectrum is confirmed by the photoluminescence measurement shown in Figure 1(b), which shows a peak wavelength of $3 \mu \mathrm{m}$ and a cut-off wavelength of $3.5 \mu \mathrm{m}$. Additionally, the spectral position of the three lasers used for characterization is marked with black, red, and blue marks for $\mathrm{CO}_{2}, \mathrm{CO}$, and $\mathrm{HCl}$, respectively. Under illumination with light wavelengths below $3.5 \mu \mathrm{m}$, electronhole pairs are created within the absorber region. Good crystal growth quality and layer thicknesses are confirmed via cross-sectional scanning electron microscopy and high-resolution x-ray diffraction measurements (both not shown here).

Circular RTD-PD devices are fabricated with photolithography techniques and dry-chemical etching. The dry-chemical etching process provides smooth sidewalls as shown in Figure 2(a). For antimony containing materials, the dry-chemical etching process induces dangling bonds due to the abrupt termination of the periodic crystalline lattice. Those create additional interfacial conductive states at the surface. ${ }^{21}$ In 
addition, oxidation of $\mathrm{Ga}(\mathrm{InAs}) \mathrm{Sb}$ gives rise to leakage currents through the formation of a semi-metallic conductive layer of $\mathrm{Sb}$ at the surface. ${ }^{29}$ Therefore, a $\mathrm{Si}_{3} \mathrm{~N}_{4} / \mathrm{SiO}_{2}$ passivation is applied for sidewall leakage reduction. An ohmic Ti/Pt/Au contact is deposited on the top and complemented by an $\mathrm{AuGe} / \mathrm{Ni} / \mathrm{Au}$ contact at the back of the sample, which is shown in the scanning electron microscopy picture in Figure 2(b) for the top contact. Electrical characterization and photocurrent measurements are performed at room temperature $\left(23^{\circ} \mathrm{C}\right)$.

Figure 3(a) and (b) shows current density-voltage characteristics of RTD-PDs with different mesa diameters of $\mathrm{d}=43,78$, and $112 \mu \mathrm{m}$ for negative and positive bias voltages, respectively. Regarding negative bias voltages, current resonances and negative differential resistance (NDR) regions are observable. At positive bias voltages, a current resonance and NDR region is observable for voltages above $3 \mathrm{~V}$ and diode diameters below $15 \mu \mathrm{m}$. The enhanced current density at positive bias voltages (compared to negative bias voltages) leads to a breakdown of the RTD due to Joule heating and disables the observation of current resonances and NDR regions for larger diode diameters. For the measurements within this paper, we therefore refrained from operating the RTDs within this possible lethal voltage region. Thicker tunneling barriers and larger ring contact areas may enable the observation of the resonance and NDR region also for large diode diameters. In the NDR regions between -0.8 to $-0.6 \mathrm{~V}$, plateaus in the current densities are observed due to the averaging of circuit oscillations. In the positive bias voltage range, the current grows exponentially without a clear resonance and NDR region. Figure 3(c) and (d) show the peak currents and valley currents and the corresponding PVCRs as a function of the diode area, respectively. The peak and valley currents increase linearly with the diode area. The PVCR saturates at a maximum value of 4.3 for large diode diameters. The linearity of the peak and valley currents as well as the saturation of the PVCR for larger RTD-PD areas indicate a good process quality with low sidewall leakage current contributions. ${ }^{30}$ The PVCR presented here is higher as the previous reported PVCR of 2.4 with electron injection from a ternary compound prewell ${ }^{31}$ This is attributed to the enhanced $\Gamma$-L valley energy separation and effective larger barrier thickness for the electron injection from the quaternary absorption layer. ${ }^{32}$

Photocurrent measurements are carried out using three DFB-Lasers with emission wavelengths of 2004 $\mathrm{nm}, 2330 \mathrm{~nm}$, and $3395 \mathrm{~nm}$ that were designed for the detection of $\mathrm{CO}_{2}, \mathrm{CO}$, and $\mathrm{HCl}$ respectively. Photocurrent measurements are performed in pulsed-mode-operation with a lock-in amplifier. The responsivity is determined from the photocurrent, the light power density, and the detector area. In order 
to measure the light power density, a beam profile was recorded prior to the experiments and a Gaussian fit was calculated. Figure 4(a) shows the absolute responsivity-voltage characteristics of the three different wavelengths for an RTD-PD with an optical diameter of $d=112 \mu \mathrm{m}$. The corresponding power on the RTD-PD is $810 \mu \mathrm{W}(2004 \mathrm{~nm}), 965 \mu \mathrm{W}(2330 \mathrm{~nm})$, and $779 \mu \mathrm{W}$ (3395 nm). Current oscillations within the NDR region are reflected by the seemingly noisy signal in the responsivity-voltage characteristic at around -0.6 to $-0.8 \mathrm{~V}$ (for an illumination wavelength of $3395 \mathrm{~nm}$ ). For all three wavelengths, the responsivity is enhanced for positive bias voltages compared to negative bias voltages by one order of magnitude. Regarding positive bias voltages, the responsivity peaks at voltage values of $0.56 \mathrm{~V}, 0.56 \mathrm{~V}$, and $1.60 \mathrm{~V}$ for $\lambda=2004 \mathrm{~nm}, \lambda=2330 \mathrm{~nm}$, and $\lambda=3395 \mathrm{~nm}$, respectively, and declines for higher voltages. The enhanced responsivity for positive bias voltages and the fact that the responsivity exhibits its maximum at intermediate voltages are clear signatures of hole accumulation near the RTS. ${ }^{24,25,32}$ The photogenerated holes which accumulate at the RTS cause a modulation of the electrostatic field and hence an additional voltage drop across the RTS emerges. This process alters the tunnelling current and leads to the observed photocurrent. Because of their low energy, incoming photons are only absorbed within the low-bandgap absorption region. Accordingly, accumulation of photogenerated holes is only achieved at positive bias voltages. At negative bias voltages, no hole accumulation occurs and therefore the photoresponse is greatly diminished. ${ }^{11,33,34,35}$ Responsivity values reach maximum values of $0.97 \mathrm{~A} / \mathrm{W}(\lambda=2004 \mathrm{~nm}), 0.74 \mathrm{~A} / \mathrm{W}(\lambda=2330 \mathrm{~nm})$, and $6.3 \mathrm{~mA} / \mathrm{W}(\lambda=3395 \mathrm{~nm})$. Because of the larger absorption coefficient, the highest responsivity is measured for the lowest incident wavelength. The maximum in the responsivity-voltage characteristics at $1.6 \mathrm{~V}$ for $\lambda=3395 \mathrm{~nm}$ is also present for the lower incident wavelengths, as can be seen in Figures 4(b) and (c). These show the photocurrent-voltage characteristic for $2004 \mathrm{~nm}$ and $2330 \mathrm{~nm}$ at different incident light powers ranging from 7 to $154 \mu \mathrm{W}$. At around $0.4 \mathrm{~V}$ and $1.4 \mathrm{~V}$ the photocurrents show two distinct peaks. The first peak decreases faster than the second one with decreasing light power. As shown in Figure 4(a), the first peak also appears for 3395 $\mathrm{nm}$ at $0.4 \mathrm{~V}$, but in a much more attenuated shape compared to the other two wavelengths. We assume that the two peaks occur due to the interplay of the bias dependent quantum efficiency ${ }^{26}$ and the hole life time at the double barrier whose interplay interdependence is not yet fully understood. With increasing light power and voltage the photocurrent increases up to maximum photocurrents of $83 \mu \mathrm{A}(\lambda=2004 \mathrm{~nm})$ and $125 \mu \mathrm{A}(\lambda=2330)$ around $0.4 \mathrm{~V}$. With higher voltages, the photocurrent decreases because of an increasing hole escape rate at the double barrier. A quantum efficiency of $\eta=0.293$ and hence a multiplication factor of 1.67 can be estimated by taking into account absorption within the absorption layer (absorption coefficient $\alpha=3 \times 10^{4} \frac{1}{\mathrm{~cm}}$, length $1=200 \mathrm{~nm}$ ) and the reflection losses at the 
semiconductor-air interface (refractive index of $\mathrm{GaSb} \sim 3.9$ ). The estimated values do not consider further loss mechanisms such as non-radiative or surface recombination. In order to determine the optimal operation voltage, the photocurrent is investigated as a function of the incident light power (see Fig. 5). The RTD-PD with a diameter $\mathrm{d}=112 \mu \mathrm{m}$ is illuminated with a wavelength of $\lambda=2330 \mathrm{~nm}$. For the two peak voltage values $(0.56 \mathrm{~V}$ and $1.6 \mathrm{~V})$, the photocurrent-light power characteristics show a nearly linear response. The two photocurrent traces intersect at around light powers of $30 \mu \mathrm{W}$. Concerning smaller and larger light powers, the second peak leads to higher respectively lower photocurrents compared to the first peak. For low bias voltages, the voltage depended quantum efficiency of RTD-PDs causes a reduction of the photocurrent via radiative electron-hole recombination in the absorber region and hence a reduction of the hole accumulation density and photocurrent. ${ }^{25}$

In summary, we have investigated RTD-PDs with GaAsSb/AlAsSb double barrier structures integrated with a quaternary $\mathrm{Ga}_{0.64} \mathrm{In}_{0.36} \mathrm{As}_{0.33} \mathrm{Sb}_{0.67}$ absorption layer covering the fingerprint absorption spectral region of $\mathrm{CO}_{2}, \mathrm{CO}$, and $\mathrm{HCl}$ in the mid-infrared wavelength region between 2 and $3.5 \mu \mathrm{m}$. The incorporation of the quaternary absorption layer into the RTD-PD enables the detector to be light sensitive up to the absorption lines of $\mathrm{HCl}$ at $3395 \mathrm{~nm}$ with a responsivity of $6.3 \mathrm{~mA} / \mathrm{W}$. At the $2004 \mathrm{~nm}\left(\mathrm{CO}_{2}\right)$ and $2330 \mathrm{~nm}(\mathrm{CO})$ absorption lines, the RTD-PD show responsivities of $0.97 \mathrm{~A} / \mathrm{W}$ and $0.74 \mathrm{~A} / \mathrm{W}$, respectively. The presented RTD-PD may pave the way towards high sensitive mid-infrared detector concepts used in tunable laser based absorption spectroscopy applications. By controlling the mole fraction of the quaternary GaInAsSb absorption layer the cut-off wavelength can be adjusted in a wide range.

The authors are grateful for financial support by the BMBF via the national project HIRT (FKZ: 13XP5003A). 

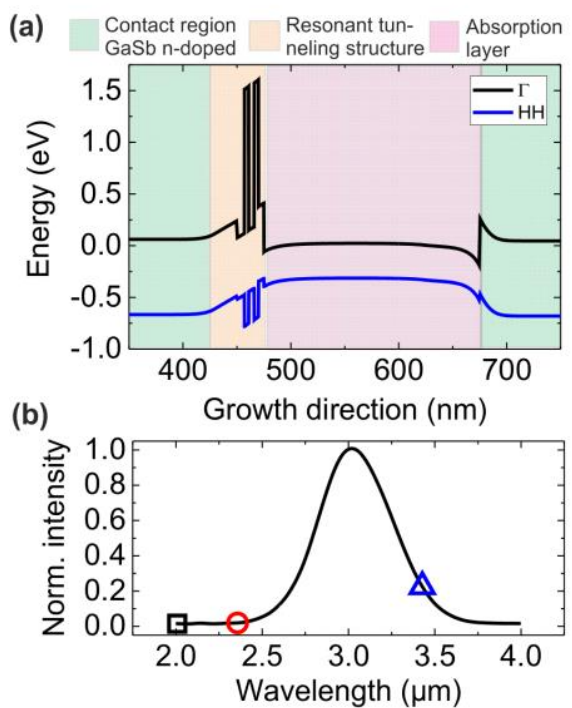

Fig. 1. (a) Numerical simulation of the bandprofile of the studied RTD-PD. The quaternary GaInAsSb absorption layer has a thickness of $200 \mathrm{~nm}$ and is embedded between the top n-doped GaSb contact layer and the RTS. Under illumination, photo-generated holes accumulate at the RTS which alter the tunneling current. (b) The photoluminescence spectrum of the absorption layer verifies the cut-off wavelength of the studied sample to be $3.5 \mu \mathrm{m}$ with a FWHM of $170 \mathrm{meV}$. The symbols mark the emission wavelengths of the three DFB-Lasers used in this study for probing the electro-optical characteristics of the RTD-PDs at $2004 \mathrm{~nm}, 2330 \mathrm{~nm}$, and $3395 \mathrm{~nm}$. 


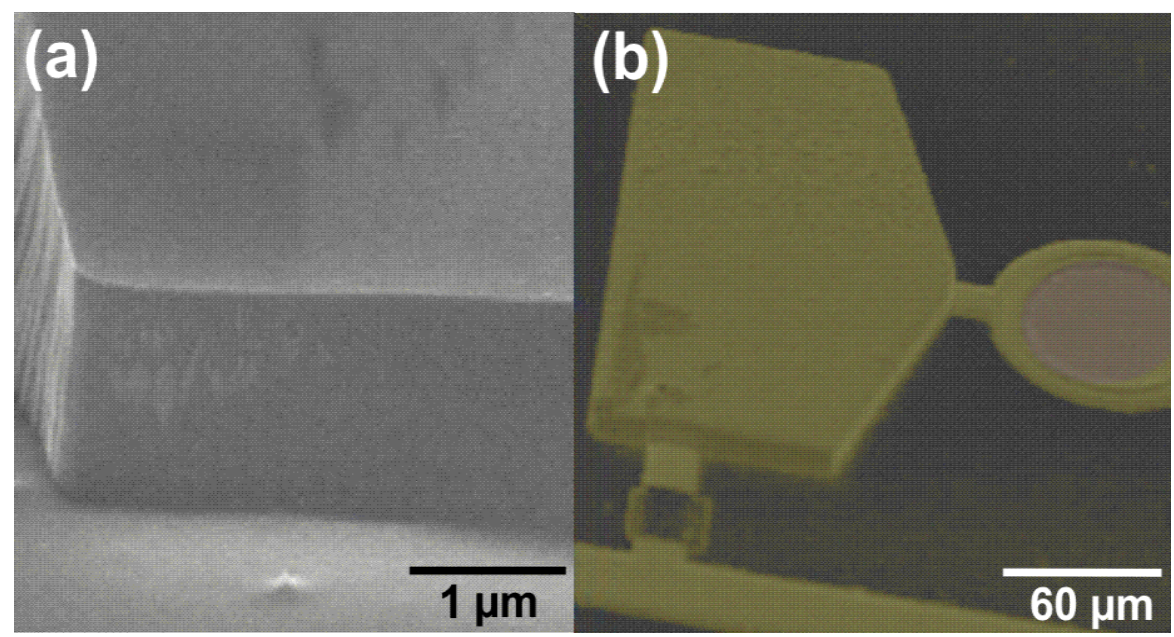

Fig. 2. Scanning electron microscopy images of the sample show the smooth sidewalls (a) and the corresponding top contact layer of the RTD-PD (b). 

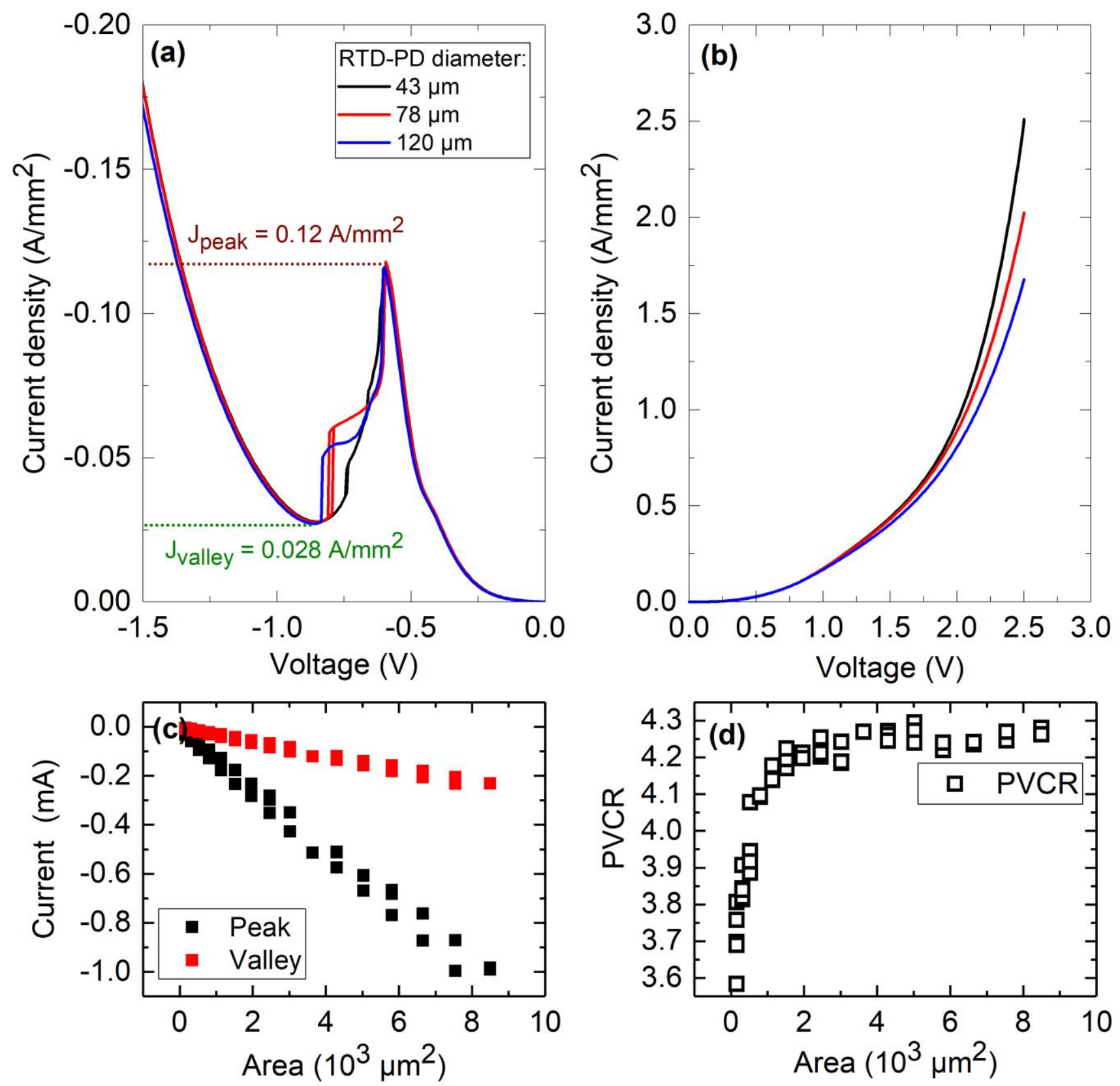

Fig 3. Current density-voltage characteristics of RTD-PDs with different mesa diameters for positive and negative bias voltages, in (a) and (b), respectively. For negative bias voltages, a resonance and NDR region are observed. At positive bias voltages, the NDR region is absent. (c) Peak currents, valley currents, and corresponding PVCR (d) of the RTD-PDs as a function of the diode area. The peak and valley currents increase linearly with the diode area. The PVCR reaches maximum values of 4.3 for large diode areas. 

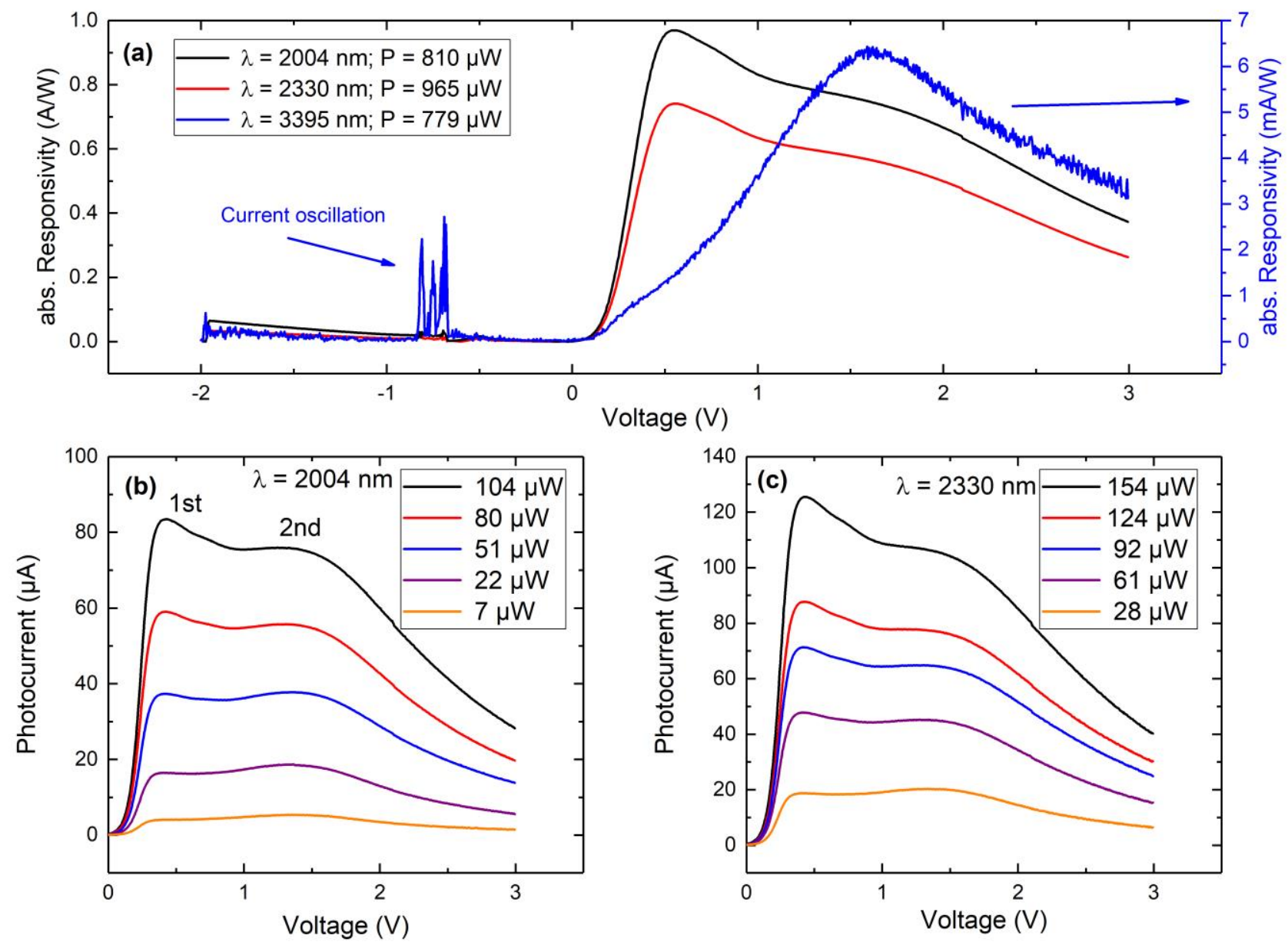

Fig. 4. (a) Absolute value of the RTD-PD responsivity for the three different laser wavelengths $(\lambda=2004$, 2330 , and $3395 \mathrm{~nm}$ ) as a function of the applied bias voltage. The laser output powers are $810 \mu \mathrm{W}, 965$ $\mu \mathrm{W}$ and $779 \mu \mathrm{W}$ for $2004 \mathrm{~nm}, 2330 \mathrm{~nm}$, and $3395 \mathrm{~nm}$ respectively. The optically active diameter of the RTD-PD is $d=112 \mu \mathrm{m}$. The responsivity is enhanced for positive bias voltages compared to negative ones and reach maximal values of $0.97 \mathrm{~A} / \mathrm{W}, 0.74 \mathrm{~A} / \mathrm{W}$, and $6.3 \mathrm{~mA} / \mathrm{W}$ for $\lambda=2004,2330$ and $3395 \mathrm{~nm}$, respectively. The current oscillations within the NDR region are well pronounced for $\lambda=3395 \mathrm{~nm}$ in the bias region around -0.7 V. RTD-PD photocurrent (in (b) and (c)) for $\lambda=2004 \mathrm{~nm}$ and $\lambda=2330 \mathrm{~nm}$ as a function of applied bias voltage, measured for six different powers. For smaller powers, the first peak (marked with $1^{\text {st }}$, at around $0.4 \mathrm{~V}$ ) diminishes, but the second one becomes more pronounced. Maximum photocurrents are obtained at the first peak. Larger voltages lead to a reduction of the photocurrent due to the increasing hole escape rate at the tunneling structure. 


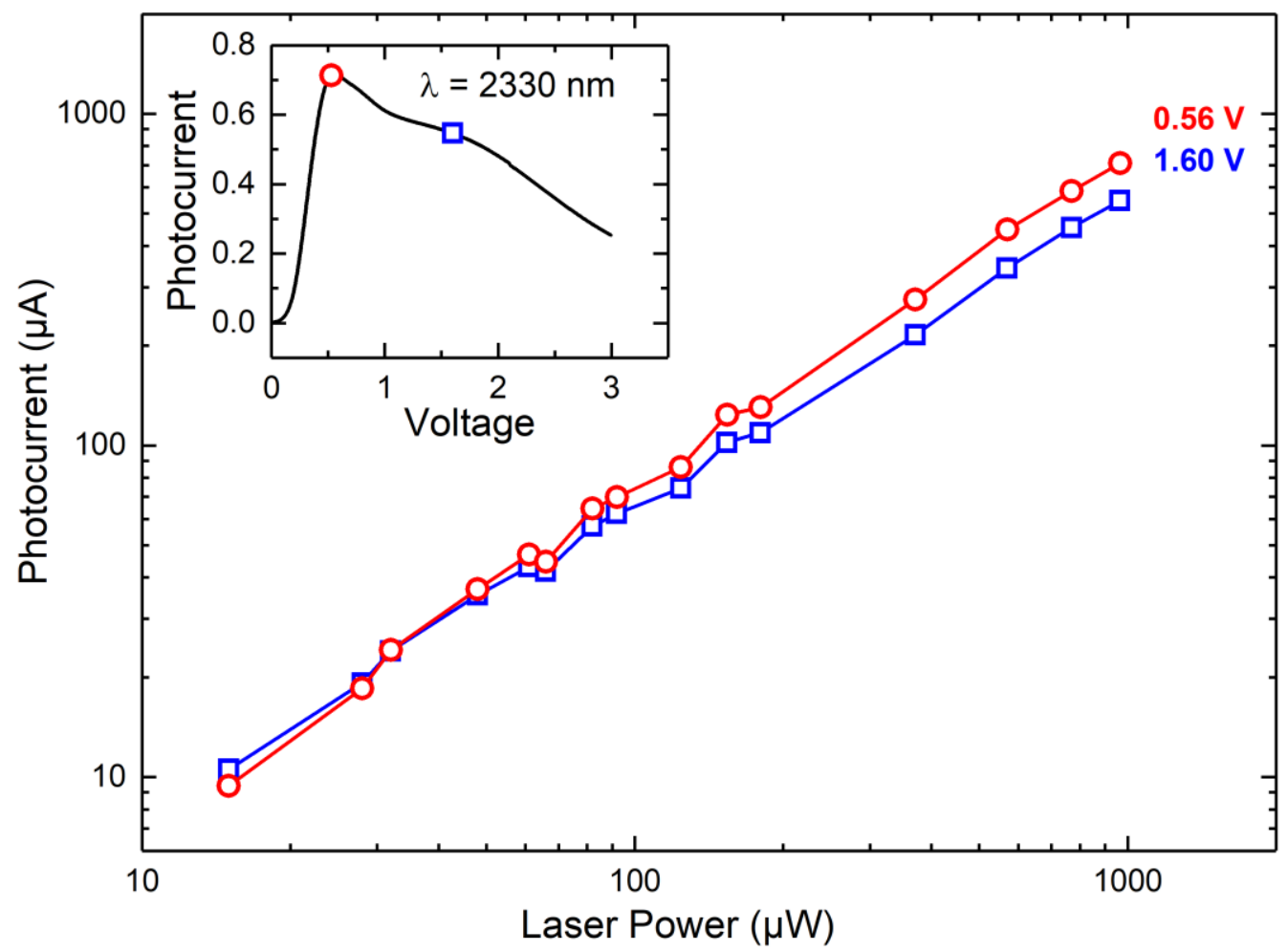

Fig. 5. Photocurrent-light power relation for bias voltages of $0.56 \mathrm{~V}$ and $1.6 \mathrm{~V}$ (see inset) for laser powers ranging from $7 \mu \mathrm{W}$ to $965 \mu \mathrm{W}$. The photocurrent traces are color coded (see inset for the corresponding photocurrent-voltage position). The two photocurrent traces intersect at around $30 \mu \mathrm{W}$ and increase linearly over the whole power range. 
${ }^{1}$ R. Tsu, Appl. Phys. Lett. 22, 562 (1973).

${ }^{2}$ L.L. Chang, L. Esaki, and R. Tsu, Appl. Phys. Lett. 24, 593 (1974).

${ }^{3}$ K. Shono, H. Kawano, T. Yokota, and M. Gomi, Appl. Phys. Express 1, 55002 (2008).

${ }^{4}$ H. Ikeda, M. Iwasaki, Y. Ishikawa, and M. Tabe, Appl. Phys. Lett. 83, 1456 (2003).

${ }^{5}$ W.S. Choi, S.A. Lee, J.H. You, S. Lee, and H.N. Lee, Nature communications 6, 7424 (2015).

${ }^{6}$ P.J. Sun, I. Haddad, and P. Mazumder, Proceedings Of The IEEE 86, 641 (1998).

${ }^{7}$ S. Suzuki, M. Asada, A. Teranishi, H. Sugiyama, and H. Yokoyama, Appl. Phys. Lett. 97, 242102 (2010).

${ }^{8}$ T. Maekawa, H. Kanaya, S. Suzuki, and M. Asada, Appl. Phys. Express 9, 24101 (2016).

${ }^{9}$ C.N. Ironside, J.M.L. Figueiredo, B. Romeira, T.J. Slight, L. Wang, and E. Wasige, in OPTO, San Francisco, California, Saturday 23 January 2010, edited by M. Razeghi, R. Sudharsanan, and G.J. Brown (SPIE, 2010.), 76080I-76080I-9.

${ }^{10}$ F. Hartmann, A. Pfenning, M. Rebello Sousa Dias, F. Langer, S. Höfling, M. Kamp, L. Worschech, L.K. Castelano, G.E. Marques, and V. Lopez-Richard, J. Appl. Phys. 122, 154502 (2017).

${ }^{11}$ A. Pfenning, F. Hartmann, F. Langer, M. Kamp, S. Höfling, and L. Worschech, in SPIE Optical Engineering + Applications, San Diego, California, United States, Sunday 28 August 2016, edited by M. Strojnik (SPIE, 2016.), p. 997307.

${ }^{12}$ J.C. Blakesley, P. See, A.J. Shields, B.E. Kardynał, P. Atkinson, I. Farrer, and D.A. Ritchie, Phys. Rev. Lett. 94, 67401 (2005).

${ }^{13}$ R.H. Hadfield, Nature Photonics 3, 696 EP - (2009).

${ }^{14}$ I.E. Gordon, L.S. Rothman, C. Hill, R.V. Kochanov, Y. Tan, P.F. Bernath, M. Birk, V. Boudon, A. Campargue and K.V. Chance et al, Journal of Quantitative Spectroscopy and Radiative Transfer 203, 3 (2017).

${ }^{15}$ A. Bauer, K. Rößner, T. Lehnhardt, M. Kamp, S. Höfling, L. Worschech, and A. Forchel, Semicond. Sci. Technol. 26, 14032 (2011).

${ }^{16}$ I.Vurgaftman, R. Weih, M. Kamp, J.R. Meyer, C.L. Canedy, C.S. Kim, M. Kim, W.W. Bewley, C.D. Merritt, J. Abell, and S. Höfling, J. Phys. D: Appl. Phys. 48, 123001 (2015).

${ }^{17}$ A. Rogalski, Progress in Quantum Electronics 27, 59 (2003).

${ }^{18}$ A. Rogalski, Infrared Physics \& Technology, 187 (2002).

${ }^{19}$ A. Rogalski, Infrared Physics \& Technology 54, 136 (2011).

${ }^{20}$ H. Lotfi, L. Li, H. Ye, R.T. Hinkey, L. Lei, R.Q. Yang, J.C. Keay, T.D. Mishima, M.B. Santos, and M.B. Johnson, Infrared Physics \& Technology 70, 162 (2015).

${ }^{21}$ W. Pusz, A. Kowalewski, P. Martyniuk, W. Gawron, E. Plis, S. Krishna, and A. Rogalski, Opt. Eng 53, 43107 (2014).

${ }^{22}$ E. Plis, M.N. Kutty, and S. Krishna, Laser \& Photonics Reviews 7, 45 (2013).

${ }^{23}$ Hossein Lotfi, Lu Li, Lin Lei, Yuchao Jiang, Rui Q. Yang, John F. Klem, and Matthew B. Johnson, Journal of applied physics 119, 023105-1 023105-8 (2016).

${ }^{24}$ S. Höfling, A. Pfenning, R. Weih, A. Ratajczak, F. Hartmann, G. Knebl, M. Kamp, and L. Worschech, in SPIE Optical Engineering + Applications, San Diego, California, United States, Sunday 28 August 2016, edited by M. Strojnik (SPIE, 2016.), p. 997306.

${ }^{25}$ A. Pfenning, F. Hartmann, M. Rebello Sousa Dias, F. Langer, M. Kamp, L.K. Castelano, V. Lopez-Richard, G.E. Marques, S. Höfling, and L. Worschech, Appl. Phys. Lett. 107, 81104 (2015).

${ }^{26}$ A. Pfenning, G. Knebl, F. Hartmann, R. Weih, A. Bader, M. Emmerling, M. Kamp, S. Höfling, and L. Worschech, Appl. Phys. Lett. 110, 033507 (2017).

${ }^{27}$ A. Pfenning, G. Knebl, F. Hartmann, R. Weih, M. Meyer, A. Bader, M. Emmerling, L. Worschech, and S. Höfling, Appl. Phys. Lett. 111, 171104 (2017).

${ }^{28}$ S. Birner, T. Zibold, T. Andlauer, T. Kubis, M. Sabathil, A. Trellakis, and P. Vogl, IEEE Trans. Electron Devices 54, 2137 (2007).

${ }^{29}$ A. Salesse, A. Joullié, P. Calas, J. Nieto, F. Chevrier, Y. Cuminal, G. Ferblantier, and P. Christol, phys. stat. sol. (c) 4, 1508 (2007).

${ }^{30}$ J. Ling, University of Rochester, Rochester, 1999.

${ }^{32}$ A. Pfenning, Hartmann F., F. Langer, M. Kamp, Höfling S., and L. Worschech, Nanotechnology 27, 355202 (2016).

${ }^{33}$ P.W. Park, H.Y. Chu, S.G. Han, Y.W. Choi, G. Kim, and E.-H. Lee, Appl. Phys. Lett. 67, 1241 (1995).

${ }^{34}$ J.F. Martins-Filho, I.J.S. Coêlho, J.M.L. Figueiredo, and C.N. Ironside, Journal of applied physics 95, 8258 (2004).

${ }^{35}$ A. Pfenning, F. Hartmann, F. Langer, M. Kamp, S. Höfling, and L. Worschech, in SPIE Optical Engineering + Applications, San Diego, California, United States, Sunday 9 August 2015, edited by M. Strojnik Scholl and G. Páez (SPIE, 2015.), p. 960810. 